\title{
ESTUDO DE COMPOTAS E DOCES CRISTALIZADOS ELABORADOS COM DIFERENTES ALBEDOS CÍTRICOS
}

\author{
ROSSANA CATIE BUENO DE GODOY * \\ EDNEIDE LUCIANA SANTIAGO MATOS ** \\ ALEXANDRA PEREIRA DOS SANTOS*** \\ TATIANE DA SILVA AMORIN****
}

\begin{abstract}
Este trabalho teve por objetivo a avaliação físico-química e sensorial de diferentes albedos cítricos, processados de maneira artesanal na forma de compotas e de produtos cristalizados. As compotas analisadas apresentaram diferença significativa, principalmente, nos valores de $\mathrm{pH}$, sólidos solúveis totais e vitamina "C". Nos produtos cristalizados, as principais diferenças ocorreram nos teores de sólidos solúveis totais, acidez total titulável e vitamina "C". Nos testes de preferência, tanto a compota quanto o produto cristalizado, elaborados com o albedo de Citrus karnas atingiram maiores médias. Conclui-se que a elaboração de compostos com albedos críticos exige que as matérias-primas sejam testadas, pois respondem de maneiras diferentes quando submetidas ao mesmo processo. Dentre as espécies estudadas, os albedos de Citrus karnas obtiveram melhor desempenho nos testes sensoriais e exigiram menor tempo de secagem. A manutenção dos albedos durante quatro dias em solução de $\mathrm{NaCl}$ não foi suficiente para a remoção de naringina, sendo necessário prolongar ou revisar essa etapa do processo. A opção pela fermentação como pré-tratamento das cascas poderá conferir ao produto cristalizado textura mais macia.
\end{abstract}

PALAVRAS-CHAVE: DOCES CRISTALIZADOS; COMPOTAS; ALBEDOS CÍTRICOS.

* Engenheiro Agrônomo, pesquisadora da EMBRAPA/CNPMF, Laboratório de Ciência e Tecnologia de Alimentos, Mandioca e Fruticultura; Cruz das Almas -BA (e-mail: catie@cnpmf.embrapa.br).

** Estudante de Agronomia da Universidade Federal da Bahia (UFBA), Bolsista da Fundação de Apoio à Pesquisa do Estado da Bahia (FAPESB).

*** Estudante de agronomia da UFBA; Bolsista da FAPESB.

**** Assistente do Laboratório Ciência e Tecnologia de Alimentos da EMBRAPA/ CNPMF. 


\section{INTRODUÇÃO}

O Brasil é o maior produtor de frutas cítricas e o maior exportador de sucos cítricos, atividade que demanda em torno de $80 \%$ da produção nacional de laranja.

O resíduo da produção industrial de suco de laranja, rico em pectina, ácido ascórbico e fibras constitui matéria-prima para a indústria de alimentos. No Brasil, grande parte desse resíduo tem sido utilizado para elaboração de polpa cítrica peletizada empregada como ingrediente de ração para bovinos (CARVALHO apud CORRÊA et al., 1999). O resíduo de citros permite ainda outras formas de aproveitamento (ARTHEY e ASHURST, 2001) com maior valor agregado, a exemplo do que ocorre em Minas Gerais. Naquele estado, o albedo dessas espécies é aproveitado para a confecção artesanal de doces em calda e cristalizados. O lucro com a comercialização dos doces excede em três vezes o valor obtido com a venda do produto para a indústria de suco.

Todo o processo agroindustrial, mesmo sendo artesanal, deve obedecer alguns critérios mínimos para obtenção de padrões de identificação e qualidade. A seleção da matéria-prima agrícola (ALMEIDA, SCHMIDT e GASPARINO FILHO, 1999), bastante heterogênea, é fundamental ao êxito de qualquer empreendimento agroindustrial.

Os trabalhos sobre a aptidão de matérias-primas para industrialização de doces disponíveis na literatura, geralmente, envolvem pêssegos, figos e ameixas. RASEIRA e NAKASU (1998) classificaram mais de vinte cultivares de pêssego para industrialização de acordo com o tamanho e formato do fruto, cor da película, cor e firmeza da polpa, aderência da polpa ao caroço, acidez e teor de sólidos solúveis e sabor do produto pronto. Para citros, os estudos restringem-se à análise do emprego de variedades para elaboração de suco concentrado.

O objetivo deste trabalho foi a avaliação físico-química, química e sensorial de compotas e doces cristalizados de albedos de cítricos elaborados com diferentes espécies.

\section{MATERIAL E MÉTODOS}

Os albedos utilizados neste trabalho foram escolhidos em função da 
disponibilidade de frutos no campo e por apresentarem tamanhos e espessura de casca diferentes.

As frutas foram colhidas no Banco Ativo de Germoplasma de Citros da Embrapa Mandioca e Fruticultura, no mês de março de 2004, no ponto de vez. Mediu-se o material selecionado com paquímetro quanto ao diâmetro e comprimento médio da fruta e espessura média do albedo.

No preparo das compotas seguiu-se a metodologia descrita por ALMEIDA, SCHIMIDT e GASPARINO FILHO, (1999), com alguns ajustes. Os frutos foram lavados e cortados verticalmente em quatro partes, sendo descartadas as polpas e as sementes. Os albedos passaram por abrasão mecânica e foram deixados em solução de $\mathrm{NaCl}$ a 10\% durante 4 dias para remoção da naringina (SILVA, 1995; SOUZA e BRAGANÇA, 2000). Posteriormente foram impregnados com xarope de sacarose, $15^{\circ}$ Brix por dia, até atingirem $50^{\circ}$ Brix. Testes preliminares evidenciaram que consumidores preferem compotas de laranja com maior teor de sacarose. As compotas foram acidificadas ( $\mathrm{pH} \mathrm{4)}$, submetidas ao tratamento térmico, resfriadas e deixadas em repouso por 30 dias.

$\mathrm{Na}$ elaboração do produto cristalizado seguiu-se a impregnação com xarope de sacarose até a saturação a 70Brix (JACKIX,1988). Efetuouse a remoção do excesso de calda. A secagem ocorreu em estufa de circulação forçada, na temperatura de $65^{\circ} \mathrm{C}$ até $25 \%$ de umidade final. Após a secagem, os albedos foram acondicionados em embalagens herméticas. Obteve-se a curva de secagem por pesagens constantes do produto em intervalos de duas horas até o peso final.

Os parâmetros físico-químicos e químicos envolveram $\mathrm{pH}$, sólidos solúveis totais (SST), acidez total titulável (ATT), analisados de acordo com a AOAC (1990) e vitamina C pelo método de Tillman (LEES, 1975).

Aplicou-se o teste sensorial de preferência, utilizando escala verbal e numérica de 7 pontos (1= desgostei muitíssimo a $7=$ gostei muito), segundo MORAES (1993). As amostras foram servidas a temperatura ambiente, em cabines, dispostas em pratos descartáveis devidamente codificados. Foram recrutados 30 julgadores entre funcionários, estudantes e visitantes da Embrapa Mandioca e Fruticultura, dos quais $70 \%$ não estavam habituados a consumir compotas cítricas e albedos cítricos cristalizados.

O experimento foi instalado em delineamento inteiramente casualizado 
com 4 tratamentos e 3 repetições. Os resultados foram submetidos à análise de variância e as diferenças entre médias comparadas pelo teste de Tukey a 5\% de probabilidade (PIMENTEL GOMES, 2000).

\section{RESULTADOS}

Verificou-se variação de 91,0 a 123,5 mm no diâmetro médio dos frutos, de 77,0 a 122,0 mm no comprimento e de 9,3 a 29,7 mm na espessura de albedo (Tabela 1).

\section{TABELA 1 - DIMENSÕES DOS FRUTOS DE DIFERENTES ESPÉCIES CÍTRICAS}

\begin{tabular}{l|c|c|c}
\hline Espécies cítricas & $\begin{array}{c}\text { Diâmetro do } \\
\text { fruto }(\mathrm{mm})\end{array}$ & $\begin{array}{c}\text { Comprimento } \\
\text { do fruto }(\mathrm{m} \mathrm{m})\end{array}$ & $\begin{array}{c}\text { Espessura } \\
\text { do albedo }(\mathrm{mm})\end{array}$ \\
\hline Citrus kamas & 100.5 & 103.5 & 20.7 \\
Citrus aurantium & 91.0 & 77.0 & 9.3 \\
Citrus obovodea & 108.0 & 112.5 & 12.0 \\
Yuma ponderosa & 123.5 & 122.0 & 16.7 \\
\hline
\end{tabular}

\subsection{COMPOTAS}

As compotas apresentaram variações na sua composição físico-química e química conforme dados demonstrados na Tabela 2.

Constatou-se diferença significativa para o pH, mesmo com a acidificação das compotas. Todas as compotas apresentaram $\mathrm{pH}$ maior do que $\mathrm{o}$ limite inferior para o desenvolvimento dos esporos de Clostridium botulinum (SOLER, RANDOMILLE, 1991). Tal fato demonstra que a acidificação dessas espécies (por segurança) deve ser abaixo de 4,0 para que após o equilíbrio osmótico, os valores finais de pH não ultrapassem 4,5. Os valores de ATT apresentaram comportamento similar aos obtidos para o $\mathrm{pH}$.

Os valores de SST, entre 39 e 46Brix, evidenciaram diferença significativa entre as compotas. Os albedos com menor espessura (Citrus aurantium e Citrus obovodea) revelaram maiores valores de SST, indicando a influência da espessura do albedo na impregnação do xarope. 


\section{TABELA 2 - AVALIAÇÕES FÍSICO-QUÍMICAS E QUÍMICAS DAS COMPOTAS DE ALBEDOS CÍTRICOS}

\begin{tabular}{|c|c|c|c|c|}
\hline Espécie & $\mathrm{pH}$ & $\begin{array}{l}\text { SST } \\
\text { oprix }\end{array}$ & $\begin{array}{c}\text { ATT } \\
\text { gáccítrico.100g. }{ }^{-1}\end{array}$ & $\begin{array}{c}\text { Vitaminac } \\
\text { mg ac.ascórbico. } 100 \mathrm{gg}^{-1}\end{array}$ \\
\hline Citrus kamas & $4,916 a$ & $41,867 b$ & 0,0476 & $0,717 b$ \\
\hline Citrus aurantium & $4,8,30$ a & 46,200 a & $0,047 b$ & $0,057 b$ \\
\hline Citrus obovodea & 4,940 a & 46,000 a & $0,053 a b$ & $0,830 b$ \\
\hline Yuma ponderosa & $4,507 b$ & $39,067 \mathrm{c}$ & 0,067 a & $1,153 \mathrm{a}$ \\
\hline Média Geral & $4,810^{* *}$ & $43,283^{*}$ & $0,053^{*}$ & $0,809^{* *}$ \\
\hline CV $(\%)$ & 1,33 & 2,19 & 14,32 & 9,31 \\
\hline
\end{tabular}

SST = Sólidos Solúveis Totais .

ATT $=$ Acidez total titulável.

$\mathrm{CV}=$ coeficiente de variação.

Médias seguidas pela mesma letra, nas colunas, não diferem estatisticamente entre si pelo teste de Tukey a $5 \%$ de probabilidade.

Apesar do tratamento térmico empregado, ainda restou pequena quantidade de vitamina "C" nos produtos (média 0,889 mg ácido cítrico. $100 \mathrm{~g}^{-1}$ ). Embora o produto processado tenha mantido teor residual de vitamina "C", as perdas foram altas em comparação com a matériaprima. CORRÊA et al. (1999) avaliaram albedos cítricos "in natura" e encontraram valores de 127 a $281 \mathrm{mg}$ ácido cítrico. $100 \mathrm{~g}^{-1}$.

A composição físico-química e química das caldas das compotas mostrou-se diferente em todas as características avaliadas (Tabela 3). Para o $\mathrm{pH}$, a calda da compota elaborada com a espécie Yuma ponderosa revelou-se a mais ácida e a da compota com Citrus karnas a menos ácida. Os valores de ATT pouco diferiram entre si.

Os sólidos solúveis totais foram maiores para as caldas de Citrus obovodea e Citrus aurantium, tal como nas compotas.

Os teores de vitamina "C" das caldas seguiram a mesma tendência apresentada pela compotas. Verificou-se que durante o equilíbrio osmótico 
houve migração da vitamina "C", contida nos albedos, para a calda.

\section{TABELA 3 - AVALIAÇÕES FÍSICO-QUÍMICAS E QUÍMICAS DAS CALDAS DAS COMPOTAS DE ALBEDOS CÍTRICOS}

\begin{tabular}{|c|c|c|c|c|}
\hline Espécie & $\mathrm{pH}$ & $\begin{array}{l}\text { SST } \\
\text { ofrix }\end{array}$ & $\begin{array}{c}\text { ATT } \\
\text { gácccitrico } 100 \mathrm{~g}^{-1}\end{array}$ & $\begin{array}{c}\text { VitaminaC } \\
\text { mg ac.ascórbico.100 g }\end{array}$ \\
\hline Citus kamas & 4,480 a & $43,533 \mathrm{C}$ & $0,0,30 b$ & $0,830 \mathrm{~b}$ \\
\hline Citrus aurantium & 4,390 ab & $45,200 \mathrm{~b}$ & 0,0306 & $0,930 \mathrm{~b}$ \\
\hline Citus obovodea & $4,387 b$ & $46,333 \mathrm{a}$ & 0,040 a & $1,100 \mathrm{~b}$ \\
\hline Yuma ponderosa & $4,137 \mathrm{C}$ & $38,733 \mathrm{~d}$ & $0,037 a b$ & 2,480 a \\
\hline Média Geral & $4,340^{* *}$ & $43,450^{\#+}$ & $0,035^{*}$ & $1,3355^{* *}$ \\
\hline$C . V(\%)$ & 0,82 & 0,46 & 11,66 & 9,55 \\
\hline
\end{tabular}

SST $=$ Sólidos Solúveis Totais .

ATT $=$ Acidez total titulável.

$\mathrm{CV}=$ coeficiente de variação.

Médias seguidas pela mesma letra, nas colunas, não diferem estatisticamente entre si pelo teste de Tukey a $5 \%$ de probabilidade.

Na opinião dos julgadores não houve diferença significativa na aparência e no aroma das compotas. Para cor, textura e sabor, as diferenças foram significativas, prevalecendo os albedos da espécie Citrus karnas para esses atributos.

Os albedos de Citrus aurantium foram os preferidos em termos de sabor, embora as médias não tenham diferido estatisticamente dos albedos de Citrus karnas (Tabela 4). Segundo os comentários dos julgadores, as compotas de Citrus obovodea e Yuma ponderosa apresentaram sabor residual amargo. A remoção da naringina desses albedos (BOBBIO e BOBBIO, 2003), provavelmente, não foi suficiente.

A média de todos os atributos avaliados situou-se na faixa entre 3,0 e 4,1 (desgostei ligeiramente a gostei ligeiramente). Nesse primeiro momento as compotas de albedos cítricos não foram muito apreciadas, 
embora a oferta desses produtos não seja comum na região em que 0 teste foi aplicado.

Considerando a média de todos os atributos, a compota elaborada com Citrus karnas obteve a melhor pontuação $(4,4)$.

TABELA 4 - ANÁLISE SENSORIAL DAS COMPOTAS DE ALBEDOS CÍTRICOS

\begin{tabular}{lccccc}
\hline Espécie & Aparência & Cor & Textura & Aroma & Sabor \\
\hline Citrus karnas & 4,267 a & 4,433 a & 4,800 a & 4,533 a & 4,233 a \\
Citrus aurantium & 4,100 a & 3,967 ab & 4,467 a & 4,333 a & 4,300 a \\
Citrus obovodea & 3,300 a & 3,700 ab & 2,967 b & 3,900 a & 1,833 b \\
Yuma ponderosa & 3,767 a & 3,433 b & 3,400 b & 3,733 a & 1,767 b \\
\hline Média Geral & $3,85^{\text {ns }}$ & $3,80^{*}$ & $3,90^{* *}$ & $4,125^{\text {ns }}$ & $3,033^{\text {* }}$ \\
CV (\%) & 38,69 & 36,06 & 38,03 & 31,06 & 50,17 \\
\hline
\end{tabular}

$\mathrm{CV}=$ coeficiente de variação.

Médias seguidas pela mesma letra, nas colunas, não diferem estatisticamente entre si, pelo teste de Tukey a $5 \%$ de probabilidade.

\subsection{CRISTALIZADOS}

Não houve diferença significativa entre o $\mathrm{pH}$ (Tabela 5) dos produtos cristalizados, cujos valores ficaram acima de 4,5 em todos os tratamentos. Isso não constitui necessariamente problema no caso de produtos desidratados, dado o baixo potencial de atividade de água (EIROA, 2002).

Para sólidos solúveis totais, os albedos de Citrus karnas evidenciaram maior impregnação de açúcar $(68,7 \%)$ e os de Yuma ponderosa apresentaram os menores valores $(63,8 \%)$.

Verificou-se pouca diferença entre os teores de acidez total titulável das amostras de forma semelhante ao perfil de $\mathrm{pH}$. 
Os teores de vitamina "C" $(0,720 \mathrm{mg}$ ácido ascórbico.100 g-1) ficaram próximos entre os tratamentos, porém abaixo dos valores verificados nas compotas (1,335 mg ácido ascórbico.100 $\left.\mathrm{g}^{-1}\right)$. O processo de cristalização com sucessivos aquecimentos ocasiona maiores perdas de vitamina "C".

\section{TABELA 5 - AVALIAÇÕES FÍSICO-QUÍMICAS E QUÍMICAS DOS ALBEDOS CÍTRICOS CRISTALIZADOS}

\begin{tabular}{lcccc}
\hline Espécie & $\mathrm{pH}$ & $\begin{array}{c}\text { SST } \\
{ }^{\circ} \mathrm{Brix}\end{array}$ & $\begin{array}{c}\text { ATT } \\
\text { g.ác.ítrico.100g }\end{array}$ & $\begin{array}{c}\text { VitaminaC } \\
\text { mg ac.ascórbico.100g }{ }^{-1}\end{array}$ \\
\hline Citrus kamas & $4,653 \mathrm{a}$ & $68,667 \mathrm{a}$ & $0,123 \mathrm{ab}$ & $0,720 \mathrm{ab}$ \\
Citrus aurantium & $4,476 \mathrm{a}$ & $66,000 \mathrm{~b}$ & $0,106 \mathrm{~b}$ & $0,723 \mathrm{ab}$ \\
Citrus obovodea & $4,696 \mathrm{a}$ & $65,667 \mathrm{~b}$ & $0,130 \mathrm{ab}$ & $0,586 \mathrm{~b}$ \\
Yuma ponderosa & $4,273 \mathrm{a}$ & $63,800 \mathrm{c}$ & $0,156 \mathrm{a}$ & $0,853 \mathrm{a}$ \\
\hline Média Geral & $4,525^{\mathrm{ns}}$ & $66,033^{*}$ & $0,129^{*}$ & $0,720^{*}$ \\
C.V(\%) & 3,68 & 0,81 & 10,95 & 11,59 \\
\hline
\end{tabular}

SST = Sólidos Solúveis Totais.

ATT $=$ Acidez total titulável.

$\mathrm{CV}=$ coeficiente de variação.

Médias seguidas pela mesma letra, nas colunas, não diferem estatisticamente entre si, pelo teste de Tukey a $5 \%$ de probabilidade.

Não foi verificada diferença significativa entre o aroma das amostras, assim como para a aparência, exceto para os albedos de Citrus aurantium (Tabela 6).

Para a textura, os albedos de Yuma ponderosa mostraram-se mais macios. Em processamentos futuros poderá ser empregada a fermentação como pré-tratamento dos albedos mais rígidos, conforme recomenda JACKIX (1988).

Para o atributo sabor não houve diferença entre os albedos de Citrus karnas e Citrus aurantium. Na avaliação do produto cristalizado, os albedos do Citrus karnas alcançaram as melhores médias $(4,62)$. 
O produto cristalizado atingiu médias maiores que as compotas, na faixa de 3,6 a 4,7 (desgostei ligeiramente a gostei ligeiramente), sendo a cor o atributo mais valorizado. Também foi percebido sabor residual de amargor para as espécies Citrus obovodea e Yuma ponderosa no teste sensorial dos cristalizados.

\section{TABELA 6 - ANÁLISE SENSORIAL DE ALBEDOS CÍTRICOS CRISTALIZADOS}

\begin{tabular}{lccccc}
\hline Espécie & Aparência & Cor & Aroma & Textura & Sabor \\
\hline Citrus kamas & $5,066 \mathrm{a}$ & $5,566 \mathrm{a}$ & $4,867 \mathrm{a}$ & $3,267 \mathrm{~b}$ & $4,367 \mathrm{a}$ \\
Citrus aurantium & $3,800 \mathrm{~b}$ & $4,233 \mathrm{~b}$ & $4,767 \mathrm{a}$ & $3,100 \mathrm{~b}$ & $4,067 \mathrm{ab}$ \\
Citrus obovodea & $4,933 \mathrm{a}$ & $4,800 \mathrm{ab}$ & $4,433 \mathrm{a}$ & $3,233 \mathrm{~b}$ & $2,633 \mathrm{c}$ \\
Yuma ponderosa & $4,833 \mathrm{a}$ & $4,400 \mathrm{~b}$ & $4,767 \mathrm{a}$ & $4,833 \mathrm{a}$ & $3,167 \mathrm{bc}$ \\
\hline Média Geral & $4,658^{* *}$ & $4,750^{* *}$ & $4,708^{\text {ns }}$ & $3,608^{* *}$ & $3,558^{*-}$ \\
CV $(\%)$ & 31,34 & 30,26 & 22,02 & 41,31 & 39,64 \\
\hline
\end{tabular}

$\mathrm{CV}=$ coeficiente de variação.

Médias seguidas da mesma letra, nas colunas, não diferem estatisticamente entre si, pelo teste de Tukey a $5 \%$ de probabilidade.

A perda de água concentrou-se nas primeiras 6 horas de desidratação (12 a $19 \%$ ) em todos os tratamentos efetuados (Figuras 1, 2, 3 e 4), obedecendo aos princípios da secagem. No início, a evaporação ocorre na superfície com a água difundindo-se para a superfície na mesma velocidade em que é evaporada (WEBER, 2001).

O tempo de secagem constitui fator importante a ser considerado, pois está relacionado com o custo de energia. Neste estudo, o tempo necessário para a secagem dos albedos de Citrus karnas foi de 14,0 h, para Citrus aurantium de 14,5 h, para Citrus obovodea de 47,3 h e para Yuma ponderosa de 20,0 h. Assim, os albedos de Citrus karnas e Citrus aurantium apresentaram maior viabilidade econômica.

A espessura do albedo do Citrus karnas $(20,7 \mathrm{~mm})$ não dificultou 0 processo de difusão da umidade durante a secagem, o que pode ter sido compensado pela permeabilidade do tecido vegetal à saída de água. 
FIGURA 1 - TEMPO DE SECAGEM DE ALBEDOS DE CITRUS KARNAS CRISTALIZADOS

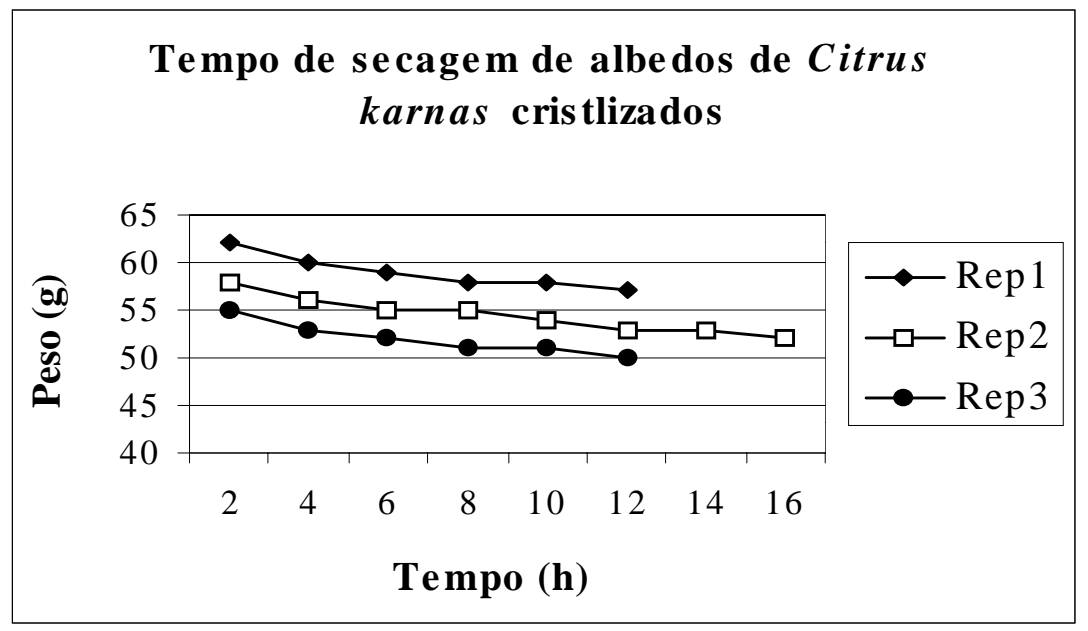

FIGURA 2 - TEMPO DE SECAGEM DE ALBEDOS DE CITRUS AURANTIUM CRISTALIZADOS

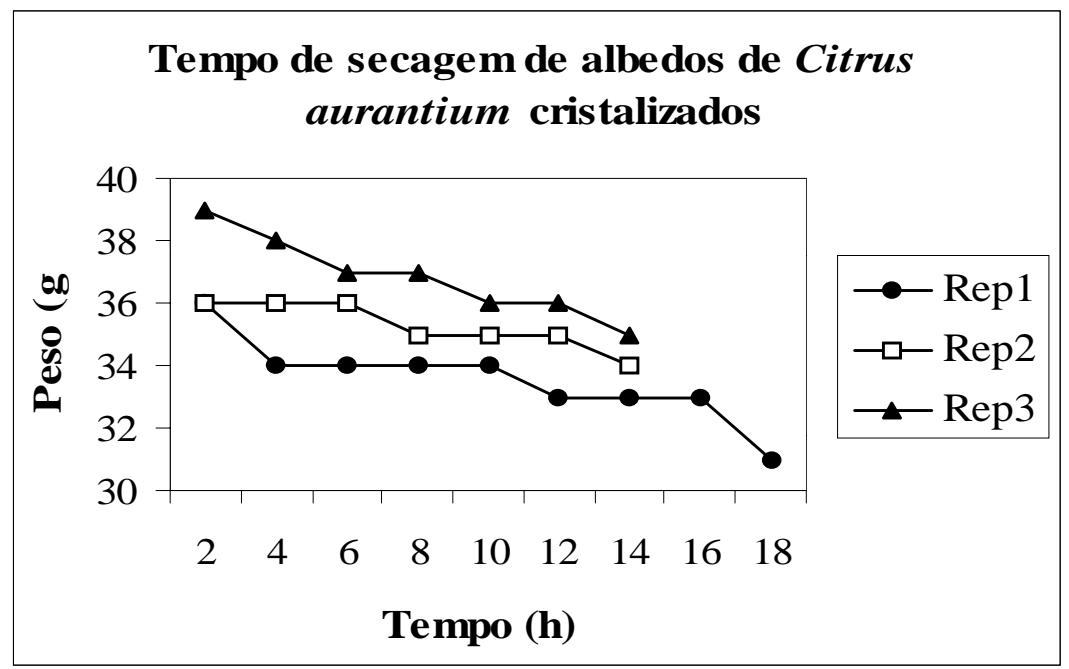


FIGURA 3 - TEMPO DE SECAGEM DE ALBEDOS DE CITRUS OBOVODEA CRISTALIZADOS

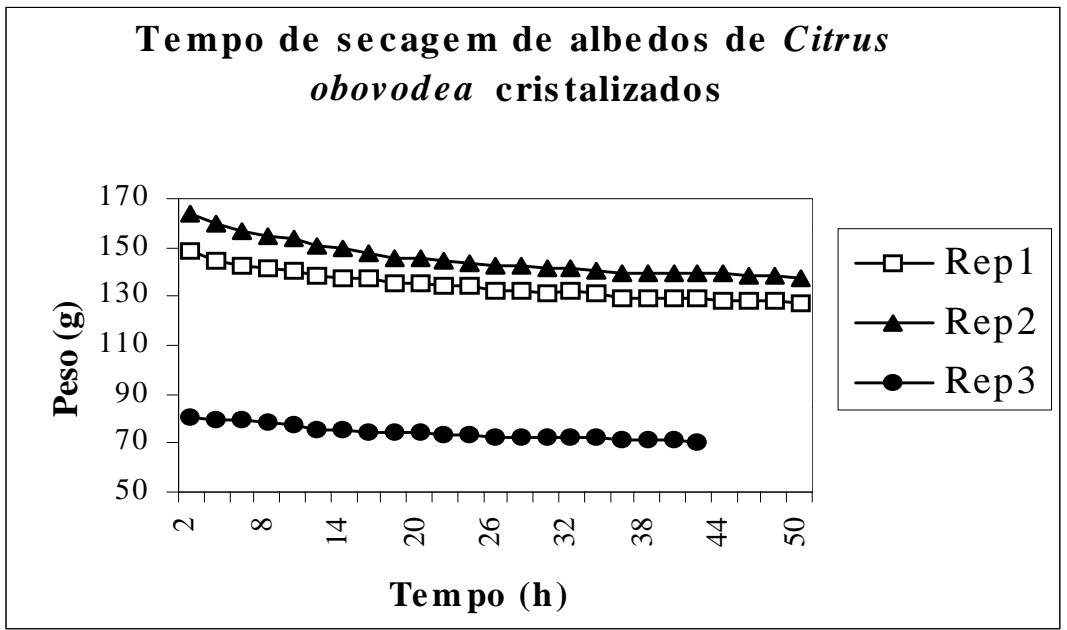

FIGURA 4 - TEMPO DE SECAGEM DE ALBEDOS DE YUMA PONDEROSA CRISTALIZADOS

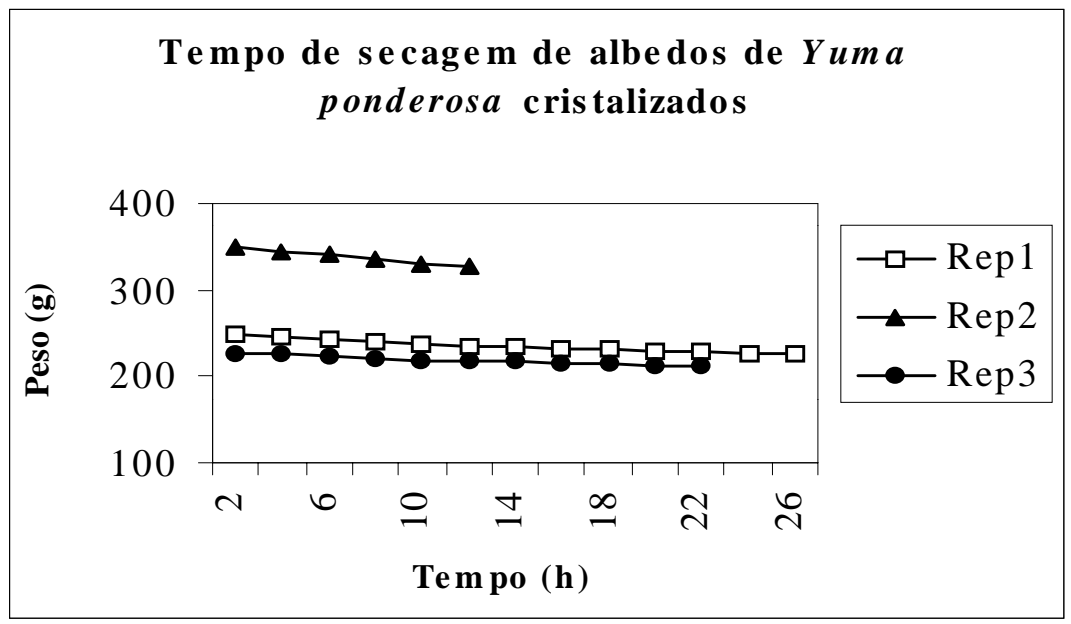




\title{
4 CONCLUSÃO
}

A elaboração de compotas com albedos cítricos exige que as matériasprimas sejam testadas a fim de padronizar a qualidade do produto final, pois respondem de maneiras diferentes quando submetidas ao mesmo processo.

Dentre as espécies estudadas, os albedos de Citrus karnas foi o que obteve melhor desempenho nos testes sensoriais para compotas e produtos cristalizados. Além disso, requer menor tempo de secagem.

A manutenção dos albedos durante quatro dias em solução de $\mathrm{NaCl}$ não foi suficiente para a remoção de naringina, sendo necessário prolongar ou revisar essa etapa do processo. A opção pela fermentação como prétratamento das cascas poderá conferir ao produto cristalizado textura mais macia.

Os testes sensoriais também devem ser aplicados em regiões em que o consumo de compotas e produtos cristalizados de albedos cítricos é habitual.

\begin{abstract}
STUDY OF CRYSTALLIZED FRUITS AND PRESERVES ELABORATED WITH DIFFERENT CITRIC ALBEDOS

This work had as objective the physical-chemical and sensorial evaluation of different citric albedos, processed in a crafty matter, in the form of fruit preserves and of dried crystallized products. The analyzed fruit preserves presented significant difference, mainly, in the $\mathrm{pH}$ values, total soluble solids and vitamin "C". In the crystallized products, the main differences occurred in the contents of total soluble solids, total titratable acidity and vitamin "C". In the preference test, the fruit preserve as well as the crystallized products elaborated with Citrus karnas albedo, reached the highest averages. In conclusion, there is a need to test raw materials once they respond differently when submitted to the same process. Among the studied species, the albedos of Citrus karnas obtained better acting in the sensorial tests for fruit preserves and crystallized products. Besides it requests smaller drying time. The maintenance of the albedos for four days in $\mathrm{NaCl}$ solution was not enough for the naringina removal, being necessary to prolong or to revise that stage of the process. The option for the fermentation as pre-treatment of the peels can confer to the crystallized product softer texture.
\end{abstract}

KEY-WORDS: CRYSTALLIZED SWEETS; FRUIT PRESERVES; CITRIC ALBEDOS. 


\section{REFERÊNCIAS}

1 ALMEIDA, M.E.M.; SCHMIDT, F.L.; GASPARINO FILHO, J. Processamento de compotas, doces em massa e geléias: fundamentos básicos. Campinas: ITAL, 1999. 61 p. (Manual técnico, 16)

AOAC. Association of Official Analytical Chemists. Official methods of analysis of AOAC Internacional. $15^{\text {th }}$ ed. Arlington, 1990. p. 912 , 1010, 1011.

ARTHEY, D.; ASHURST, P.R. Fruit processing: nutrition, products, and quality management. $2^{\text {nd }}$ ed. Gaithersburg: Aspen Publishers, 2001. $312 \mathrm{p}$.

BOBBIO, F.O.; BOBBIO, P.A. Introdução a química de alimentos. São Paulo: Varela, 2003. 238 p.

5 CORRÊA, A.D.; RIOS, A.L.O.; LOPES, L.M.V.; CONCEIÇÃO, A. Alguns constituintes químicos do albedo de frutos cítricos. Revista Brasileira de Fruticultura, Jaboticabal, v.21, n.1, p.17-19, abr. 1999.

6 EIROA, M.N.U. Microbiologia de frutas e hortaliças desidratadas. In: AGUIRRE, J.M.; GASPARINO FILHO, J. Desidratação de frutas e hortaliças. Campinas: ITAL, 2002. cap. 6. p.1-26.

JACKIX, M. H. Doces, geléias e frutas em calda. Campinas: UNICAMP, 1988. 172 p. (Série tecnologia de alimentos).

LEES, R. Food analysis and quality control methods for the food manufacturer and buyer. London: Leonard Hil Books, 1975.

9 MORAES, M.A.C. Métodos para avaliação sensorial dos alimentos. 8. ed. Campinas: UNICAMP, 1993. 93 p.

10 PIMENTEL GOMES, F. Curso de estatística experimental. 14. ed. Piracicaba: Nobel, 2000. 477 p.

11 RASEIRA, M.C.B.; NAKASU, B.H. Cultivares: descrição e recomendação. In: MEDEIROS, C.A.B; RASEIRA, M.C.B. A cultura do pessegueiro. Pelotas: CPACT, 1998. cap. 3, p.29-90.

12 SILVA, D.M.A.G. Compotas. Cuiabá: EMPAER-MT, 1995. $16 \mathrm{p}$.

13 SOLER, M.P.; RADOMILLE, L.R.; TOCCHINI, R.P. Processamento. In: SOLER, M.P.; BLEIROTH, E.W.; IADEROZA, M.; DRAETTA, I.S.; 
LEITÃO, M.F.F.; RADOMILE, L.R.; TOCCHINI, R.P.; FERREIRA, V.L.P.; MORI, E.E.M.; SOLER, R.M.; ARDITO, E.F.G.; XAVIER, R.L.; TEIXEIRA NETO, R.O. Industrialização de frutas. Campinas: ITAL, 1991. 205 p. (Manual Técnico, n.8).

14 SOUZA, C.M; BRAGANÇA, M.G.L. Manual de processamento artesanal de frutas. Belo Horizonte: EMATER-MG, 2000. 122 p.

15 WEBER, E. A. Armazenagem agrícola. Guaíba: Agropecuária, 2001. $395 \mathrm{p}$.

\section{AGRADECIMENTOS}

À Fundação de Amparo à Pesquisa do Estado da Bahia. 